\title{
Gout of hand and wrist: the value of US as compared with DECT
}

\author{
Andrea S. Klauser ${ }^{1} \cdot$ Ethan J. Halpern ${ }^{2} \cdot$ Sylvia Strobl ${ }^{1}$. Mohamed M. H. Abd Ellah ${ }^{1,3} \cdot$ Johann Gruber $^{4}$. \\ Rosa Bellmann-Weiler ${ }^{4}$. Thomas Auer ${ }^{1}$. Gudrun Feuchtner ${ }^{1} \cdot$ Werner Jaschke ${ }^{1}$
}

Received: 21 November 2017 / Revised: 25 January 2018 / Accepted: 31 January 2018 / Published online: 20 April 2018

(C) The Author(s) 2018

\begin{abstract}
Objectives The purpose of this study was to compare findings of ultrasound (US) with dual-energy CT (DECT) in patients presenting with suspected gouty hand and wrist arthritis.

Methods This prospective study included 180 patients (136 men and 44 women, age range, 31- 94 years; mean age, 65.9 years) with an initial clinical diagnosis of acute gouty arthritis who underwent DECT and US examination. Intra- and extra-articular findings of each modality were tabulated and calculated with DECT as gold standard.

Results The final diagnosis of gout was positive in $97 / 180$ patients (53.9\%) by DECT, an alternative diagnosis confirmed in 83 patients. US showed a sensitivity of $70.1 \%$ (extra-articular: $42.5 \%, p<0.0001$; intra-articular: $80.3 \%, p=0.14$ ) and specificity of $51 \%$. The double contour sign (DCS) was present in 58/61 patients with a positive US study for intra-articular gout (95.1\%).

Conclusions Sensitivity of US for diagnosis of gouty arthritis in hand and wrist is limited, particularly with respect to extraarticular urate deposition. The DCS is the most sensitive sign for the assessment of gouty hand and wrist arthritis by US.

Key points

- Sensitivity of US for diagnosis of gouty arthritis in hand and wrist is limited, particularly with respect to extra-articular gouty deposits.

- The double contour sign is the most sensitive finding for the assessment of gouty hand and wrist arthritis by US.

- Although the sensitivity of US for diagnosis of gouty hand and wrist arthritis is limited, it can be used as a first-line imaging modality in the presence of the DCS.
\end{abstract}

Keywords Arthritis $\cdot$ gouty $\cdot$ Wrist $\cdot$ Hand $\cdot$ Upper extremity $\cdot$ Tomography $\cdot$ x-ray computed

\author{
Abbreviations \\ CPPD calcium pyrophosphate dehydrate deposition \\ disease \\ DCS double contour sign \\ DECT dual-energy computed tomography \\ HADD hydroxyapatite deposition disease
}

Sylvia Strobl

Sylvia.Strobl@tirol-kliniken.at

1 Radiology Department, Medical University Innsbruck, Anichstrasse 35, 6020 Innsbruck, Austria

2 Department of Radiology and Urology, Jefferson Prostate Diagnostic and Kimmel Cancer Center, Thomas Jefferson University, 1025 Walnut Street, Suite 1112, Philadelphia, PA 19107, USA

3 Diagnostic Radiology Department, South Egypt Cancer Institute, Assiut University, Assiut 71515, Egypt

4 Department of Internal Medicine II, Medical University Innsbruck, Anichstrasse 35, 6020 Innsbruck, Austria
MSU monosodium urate

SF synovial fluid

US ultrasound

\section{Introduction}

The incidence of gout has tripled over recent decades and now represents the most common inflammatory arthritis in men and women [1-4].

Long-term hyperuricemia results in deposition of monosodium urate (MSU) crystals in joints and soft tissues, triggering gouty arthritis and, if not properly treated, the formation of gouty tophi [5-8]. With respect to imaging methods Bongartz et al. [4] showed that DECT has a significant impact on clinical decision-making when gout is suspected. In the studies by Dalbeth [9] and Zhang et al. [10], DECT is described as an advanced imaging method for longitudinal 
follow-up to monitor treatment response when looking at tophus size regression [11-13].

Few studies have evaluated the feasibility of ultrasound (US) as compared to dual-energy CT (DECT), which can differentiate calcium-rich material (high attenuation) from MSU crystal-rich material (low attenuation) for the diagnosis of gout [14-16]. Manger et al. [17] described periarticular gouty deposits in 9/12 hands and wrists, whereas Huppertz et al. [18] presented overall results for multiple joints (knees, feet, elbows and hands) without detailed sonographic results for hands and wrists in their patient-based evaluation.

Therefore the aim of our study was to evaluate US findings compared to DECT in terms of intra- and extra-articular gouty deposits in patients presenting with acute arthritis of the hand and/or wrist with suspected gout.

\section{Materials and methods}

\section{Patients}

This prospective, HIPPA-compliant study received institutional review board approval, and written informed consent was obtained from all participants.

Between 2015 and 2016, 180 patients with a history of gout fulfilling the American Rheumatism Association classification criteria [19] for gouty arthritis of the hand and/or wrist were included. The initial diagnosis was based on clinical symptoms including swelling of the wrist/hand and/or hotness, redness or tenderness for 1-4 weeks' duration, as well as serum urate levels. Patients were referred to DECT and US within 1-3 days by the rheumatology department, after being examined by a rheumatologist with 15 years of experience (ASK) [19].

A single symptomatic hand/wrist was evaluated for each study participant. Patients with history of inflammatory rheumatic disease, hand/wrist trauma or previous hand/wrist surgery were excluded.

\section{DECT examination}

All patients were referred to dual-source CT scan (Somatom Definition Flash; Siemens Healthineers) using two different energy levels ( 80 and $140 \mathrm{kV}$ ), with a previously described dual-tube protocol [14].

Scan parameters included a $2 \times 64 \times 0.625 \mathrm{~mm}$ acquisition at a rotation time of $0.33 \mathrm{~s}$. Transverse sections were reconstructed from the DE data sets, at a resolution of $0.4 \mathrm{~mm}$ using the soft tissue kernel (D30) and bone kernel (B60). Tube currents ranged from 100 to $140 \mathrm{mAs}$ for tube $\mathrm{A}$ and between 200 to $250 \mathrm{mAs}$ for tube $\mathrm{B}$, based upon automatic care dose CT software. The soft tissue kernel data sets of both tubes were loaded onto a Syngo Multi-Modality Workplace (Siemens Healthineers) and reconstructed with a commercially available software program (DE Gout; Siemens Healthineers). This software provides color-coded images in the transverse, sagittal and coronal image planes, with a slice thickness of $0.75 \mathrm{~mm}$ at a slice increment of $0.5 \mathrm{~mm}$. The CT examination extended from the distal forearm to the fingertips.

A commercially available picture archiving and communication system (PACS) was used (IMPAX; Agfa-Gevaert).

Color-coded DECT images were evaluated by a radiologist with 5 years of experience (SS), who was blinded to clinical data and US examination and who classified the findings as positive or negative for the presence of MSU deposits. A positive scan was defined as the presence of color-coded MSU deposits according to Huppertz et al. [18]. In addition, we included a detailed assessment of the location of MSU deposits to differentiate intra- and extra-articular deposits as follows:

Intra-articular: triangular fibrocartilage complex, distal radioulnar joint, radiocarpal joint, intercarpal, carpometacarpal joint, metacarpal joint, proximal interphalangeal joint, distal interphalangeal joint, erosion, and double contour sign. Extraarticular: extensor and flexor tendons, and lymphedema.

According to American College of Rheumatology (ACR)/ European League Against Rheumatism (EULAR) guidelines nail bed deposits, sub-millimetre deposits, skin deposits, and deposits obscured by motion, beam hardening and vascular artefact were not classified as positive findings in our study [20].

\section{US examination}

Sonographic examination was performed according to European Society of Musculoskeletal Radiology (ESSR) guidelines, using a 15-6 MHz linear array transducer (HI Vision Preirus, Hitachi Aloka Medical) by a musculoskeletal radiologist with 10 years of experience (ASK), blinded to DECT and clinical findings and not allowed to talk to the patient regarding further details.

A positive US diagnosis was provided in the presence of a double contour sign (DCS) or based upon visible aggregates or tophi and tendon depositions according to international consensus OMERACT (outcome measures in rheumatoid arthritis clinical trials) guidelines [21].

The DCS was characterized by an abnormal hyperechoic band over the superficial margin of the articular hyaline cartilage, independent of the angle of insonation. This band might be irregular or regular, continuous or intermittent, and could be distinguished from the cartilage interface sign and from CPPD deposits located within the cartilage [21].

A tophus was a circumscribed, inhomogeneous, hyperechoic and/or hypoechoic aggregation (which may or may not generate posterior acoustic shadow), which might be surrounded by a small anechoic rim [21].

Aggregates (which might be intra-articular or intratendinous) were heterogeneous hyperechoic foci that maintained a high 
degree of reflectivity even when the gain setting was minimized or the insonation angle was changed and which occasionally generated posterior acoustic shadow [21].

Intra-articular US findings included DCS, aggregates, tophi and erosions. For extra-articular US findings extensor and flexor tendons were evaluated.

Absence of the above described findings was tabulated as a negative US study, while any detected finding that did not match with the typical criteria of OMERACT guidelines was considered indeterminate (e.g. interrupted or very thin double contour line or hyperechoic unclear deposits, which might represent calcifications in tendons or might be caused by osteoarthritis). Indeterminate US findings were tabulated as missed by US relative to DECT.

\section{Clinical parameters}

Clinical assessment was performed in patients presenting with acute hand/wrist arthritis having a history of gout based on the American Rheumatism Association classification criteria [19]. Serum uric acid levels and CRP were determine at the time of US and DECT examination according to ACR/EULAR guidelines and were rated respectively as elevated above $6 \mathrm{mg} / \mathrm{dl}$ [20] and CRP above $0.5 \mathrm{mg} / \mathrm{dl}$. Both DECT and US operators were blinded to clinical and laboratory findings, as well as to each other's imaging findings.

\section{Statistical analysis}

Statistical analysis was performed with Stata 12.1 (StataCorp). The presence of positive findings for gout was tabulated for each location in the hand/wrist for each DECT and US. DECT and US findings were observed and recorded independently. The sensitivity and specificity of US were calculated with DECT as the gold standard. Since the US and DECT studies were performed on the same patients (paired study design), the frequency of positive findings for gout based upon US versus DECT was compared with a McNemar's chi square for symmetry, with a $p$ value of 0.05 indicating a statistically significant result. Three comparisons of frequency of positive findings for gout were performed between DECT and US: frequency of overall diagnosis, frequency of extra-articular diagnosis and frequency of intra-articular diagnosis.

\section{Results}

\section{DECT and US findings}

An overall diagnosis of gout (intra-articular and/or extra-articular) in 180 patients (136 men and 44 women, mean age, 65.9 years; age range, 31-93 years, disease duration 1-9 years, mean $5 \pm 3.4$ years) was demonstrated in 71/180 (39.4\%) of patients by US, and in 97/180 (53.9\%) of patients by DECT (McNemar chi square for symmetry, $p<0.0001$ ). Among the 83 patients with a negative DECT study, the final alternative diagnosis was confirmed as osteoarthritis in $42 / 83$ (50.6\%), calcium pyrophosphate dehydrate deposition disease (CPPD) in $31 / 83(37.3 \%)$ and hydroxyapatite deposition disease (HADD) in 10/83 (12\%). DECT and US findings were concordant for the overall diagnosis of gouty arthritis in 110/180 $(61.1 \%)$ of patients (Table 1$)$.

Among 97 patients with a positive diagnosis of gout by DECT, US was positive in $68 / 97$ patients (sensitivity = $70.1 \%$ ). Among 83 patients with a negative DECT study, US was negative in $42 / 83$ (specificity $=51 \%$ ). When indeterminate US studies were counted as negative, the specificity of US increased to $96.4 \%(80 / 83)$.

The distribution of US and DECT findings are summarized in Table 2. A comparison between US and DECT is provided for intra- and extra-articular findings in Table 1. Among 73 patients with positive extra-articular findings on DECT, US was positive for an extra-articular finding in 31 patients (sensitivity $=42.5 \%$, McNemar chi square for symmetry, $p<0.0001$ ). Among 76 patients with positive intra-articular findings on DECT, US was positive for an intra-articular finding in 61 patients (sensitivity $=80.3 \%$, McNemar chi square for symmetry, $p=0.14)$. The DCS was the most frequently positive US sign for the diagnosis of gouty hand and wrist arthritis (Figs. 1, 2 and 3). Among the 61 patients with a positive US study for intra-articular gout that was concordant with DECT, DCS was present in 58 (95.1\%). Overall sensitivity of the DCS among the 97 patients with a positive DECT study was 63/97 (sensitivity $=65 \%$ ), and among the 76 patients with a positive intraarticular DECT study was 58/76 (sensitivity $=76 \%$ ). A positive DCS was also present in 3/83 patients with a negative DECT study (specificity $=96.4 \%$ ). Thus, $63 / 66$ patients with a DCS had MSU deposits on DECT.

Table 1 Correlation between US and DECT

\begin{tabular}{|c|c|c|c|c|c|c|}
\hline & & & \multicolumn{4}{|l|}{ US } \\
\hline & & & 1 & 2 & 3 & Total \\
\hline & Total & 1 & 42 & 3 & 38 & \\
\hline & & 2 & 20 & 68 & 9 & \\
\hline \multirow[t]{6}{*}{ DECT } & Intra-articular & 1 & 58 & 8 & 38 & 104 \\
\hline & & 2 & 9 & 61 & 6 & 76 \\
\hline & & Total & 67 & 69 & 44 & 180 \\
\hline & Extra-articular & 1 & 94 & 4 & 9 & 107 \\
\hline & & 2 & 42 & 31 & 0 & 73 \\
\hline & & Total & 136 & 35 & 9 & 180 \\
\hline
\end{tabular}

1 negative findings, 2 positive findings, 3 indeterminate findings, $U S$ ultrasound,

DECT dual-energy computed tomography 
Table 2 Comparison of deposition sites between DECT and US

\begin{tabular}{llllll}
\hline & Deposition site & DECT Number of patients & $\%$ & US Number of patients & $\%$ \\
\hline Intra-articular & TFCC/DRU & $53 / 97$ & 54.6 & $34 / 97$ & 35.1 \\
& RC & $53 / 97$ & 54.6 & $34 / 97$ & 35.1 \\
& IC & $55 / 97$ & 56.7 & $34 / 97$ & 35.1 \\
& CMC & $34 / 97$ & 35.1 & $29 / 97$ & 29.9 \\
& MCP & $41 / 97$ & 42.3 & $41 / 97$ & 42.3 \\
& PIP & $32 / 97$ & 33 & $20 / 97$ & 20.6 \\
& DIP & $16 / 97$ & 16.5 & $11 / 97$ & 11.3 \\
& DCS & - & - & $66 / 97$ & 68 \\
Extra-articular & Extensor tendons & $29 / 97$ & 30 & $16 / 97$ & 16.5 \\
& Flexor tendons & $63 / 97$ & 65 & $11 / 97$ & 11.3 \\
\hline
\end{tabular}

TFCC/DRU triangular fibrocartilage complex/distal radioulnar joint, $R C$ radiocarpal joint, $I C$ intercarpal joint, $C M C$ carpometacarpal joint, $M C P$ metacarpophalangeal joint, $P I P$ proximal interphalangeal joint, $D I P$ distal interphalangeal joint

\section{Serum uric acid level}

Mean uric acid level in the study population was $7.07 \mathrm{mg} / \mathrm{dl}$ (range $1.89-15.83 \mathrm{mg} / \mathrm{dl}, \mathrm{SD} \pm 4.01 \mathrm{mg} / \mathrm{dl}$ ) and $\mathrm{CRP}$ was 4.5 $\pm 0.7 \mathrm{mg} / \mathrm{dl}$ (range $0-0.5 \mathrm{mg} / \mathrm{dl}$ ). An elevated serum uric acid was present in 71/180 (39.4\%) patients.

Mean uric acid levels were only marginally higher among patients with a positive DECT study and a final diagnosis of gout, as compared to those with a negative DECT study and an alternative confirmed diagnosis $(7.4 \mathrm{mg} / \mathrm{dl}$ versus $6.6 \mathrm{mg} / \mathrm{dl})$, $p=0.06$.

Patients with a positive US study for the diagnosis of gout did demonstrate a significantly higher uric acid level than those with a negative US study $(7.7 \mathrm{mg} / \mathrm{dl}$ versus $6.2 \mathrm{mg} / \mathrm{dl})$, $p<0.01$. Uric acid levels were higher among patients with a positive DCS compared to those without DCS $(7.7 \mathrm{mg} / \mathrm{dl}$ versus $6.7 \mathrm{mg} / \mathrm{dl}), p=0.01$.

\section{Discussion}

The DCS was the most frequently detected US sign for the diagnosis of gouty hand and wrist arthritis with a sensitivity of
$76 \%$ for intra-articular gout, with an overall sensitivity of $65 \%$ (Fig. 4). For the extra-articular compartment in terms of gouty deposits in tendons DECT was superior for delineation and characterization [22]. Our study is the first to give a detailed description of MSU depositions in both the intra- and extraarticular compartments of the hands and wrists with comparison of US to DECT.

US has been advanced as an imaging modality of choice for the diagnosis and management of gout [21, 23-25]. In fact, in addition to a positive DECT result, the DCS has been incorporated in ACR/EULAR guidelines 2015 as being the most specific sign for the diagnosis of gouty arthritis $[24,26]$.

Among patients with a positive DCS, a wide range of sensitivity for DCS has been reported, even for experienced sonographers [26-30]. However, a positive DCS and a negative DECT study might be explained also by a low concentration of MSU crystals or early disease with gouty deposits not yet visible on DECT. Thiele and Schlesinger [28] detected the DCS in $92 \%$ of 23 patients, but that study included primarily the first MTP joint and knees and only four MCP joints. Ottaviani et al. [31] reported a sensitivity of 57\% and specificity of $98 \%$ for DCS, but again with MTP joints and knees. Ogdie et al. [32] reported a pooled sensitivity for DCS of 0.83
Fig. 1 Right hand of a 77-yearold male patient. Longitudinal ultrasound (US) scan of metacarpophalangeal (MCP) joint showing double contour sign (arrows). Neither tophi nor aggregates can be seen. Dual-energy CT (DECT) of the same joint (not shown) was negative

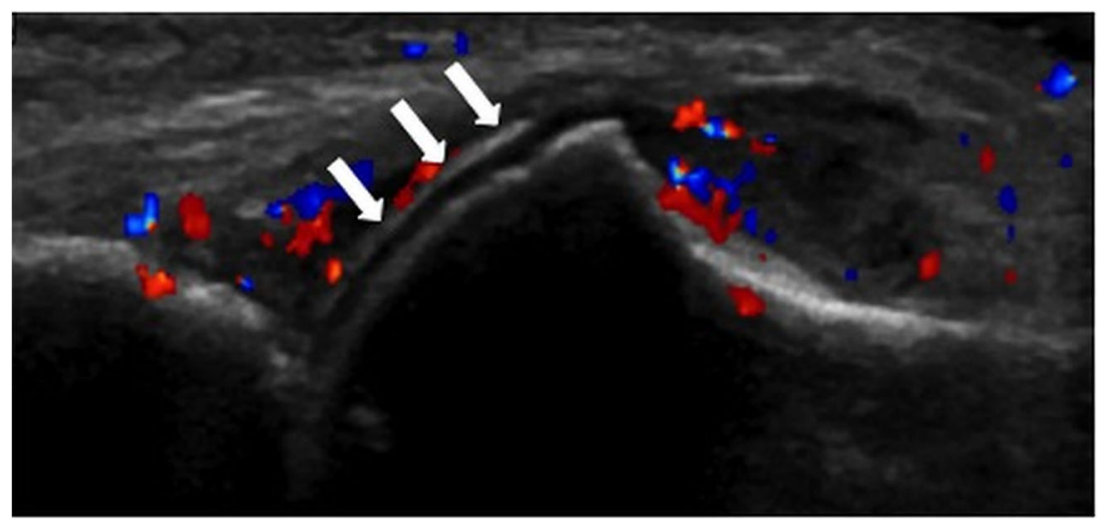




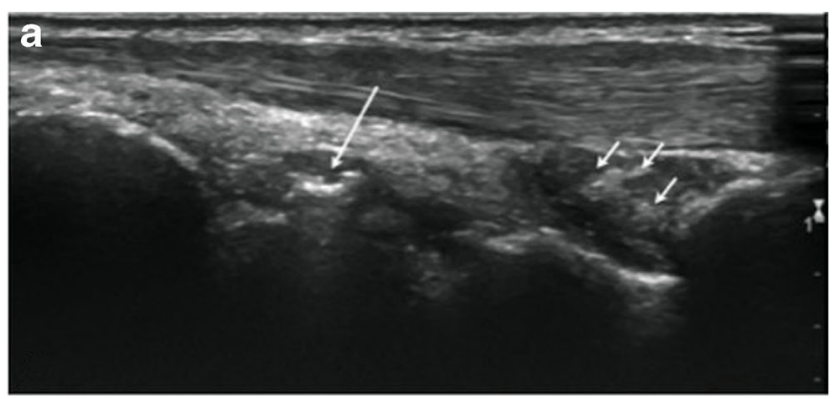

b

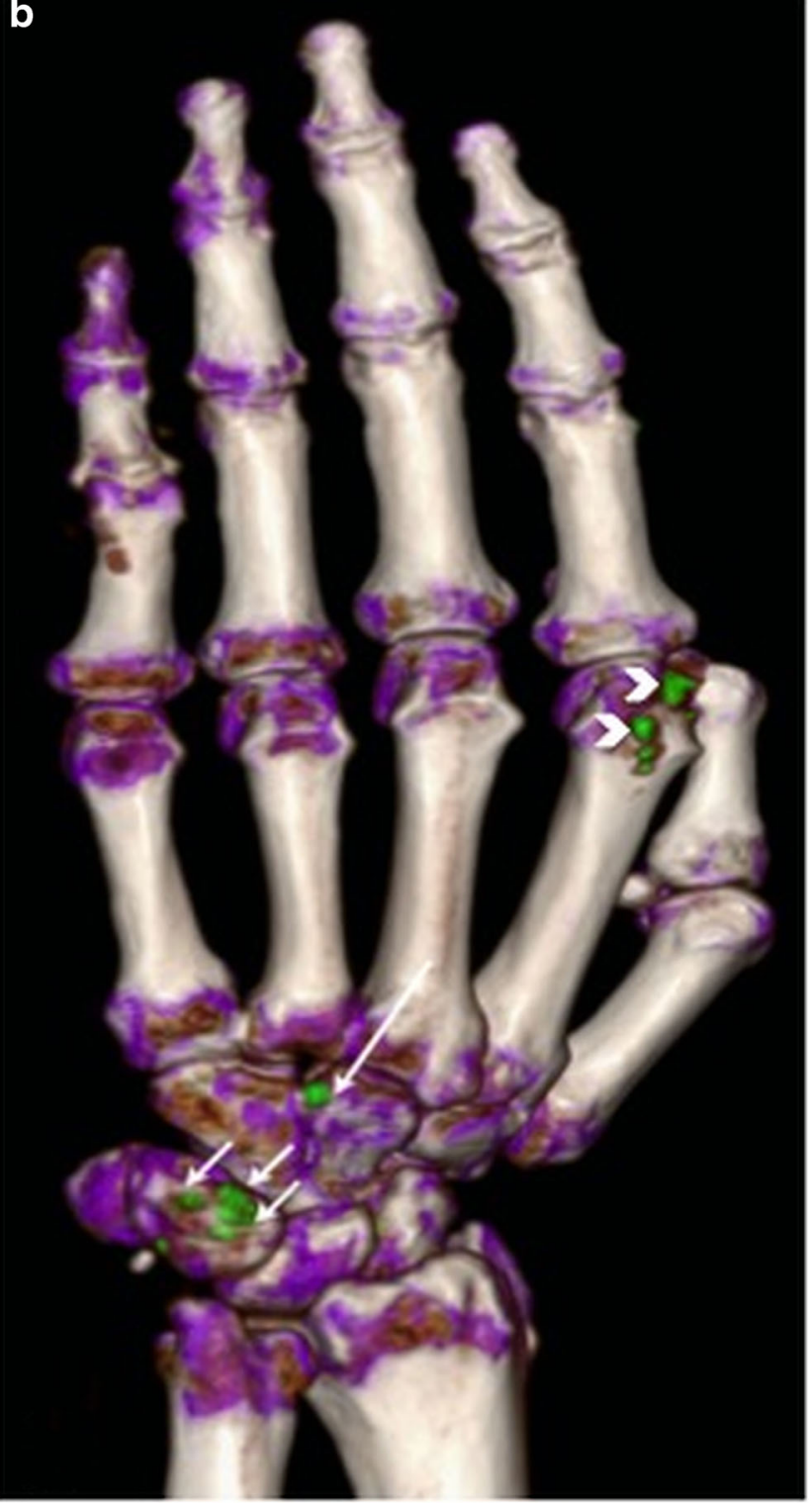

Fig. 2 Left hand of a 56-year-old female patient. a Longitudinal US scan over the dorsal wrist shows small echogenic deposits in the radiocarpal joint (short arrows) and intercarpal joint (large arrow), but no typical aggregates or tophi and was rated as indeterminate case. b DECT 3D volume rendered image of the same hand showing MSU deposits at the radiocarpal joint (short arrows) and intercarpal joint (large arrow). Note: MSU deposits at dorsal aspect of 2nd MCP joint (small white arrow heads)
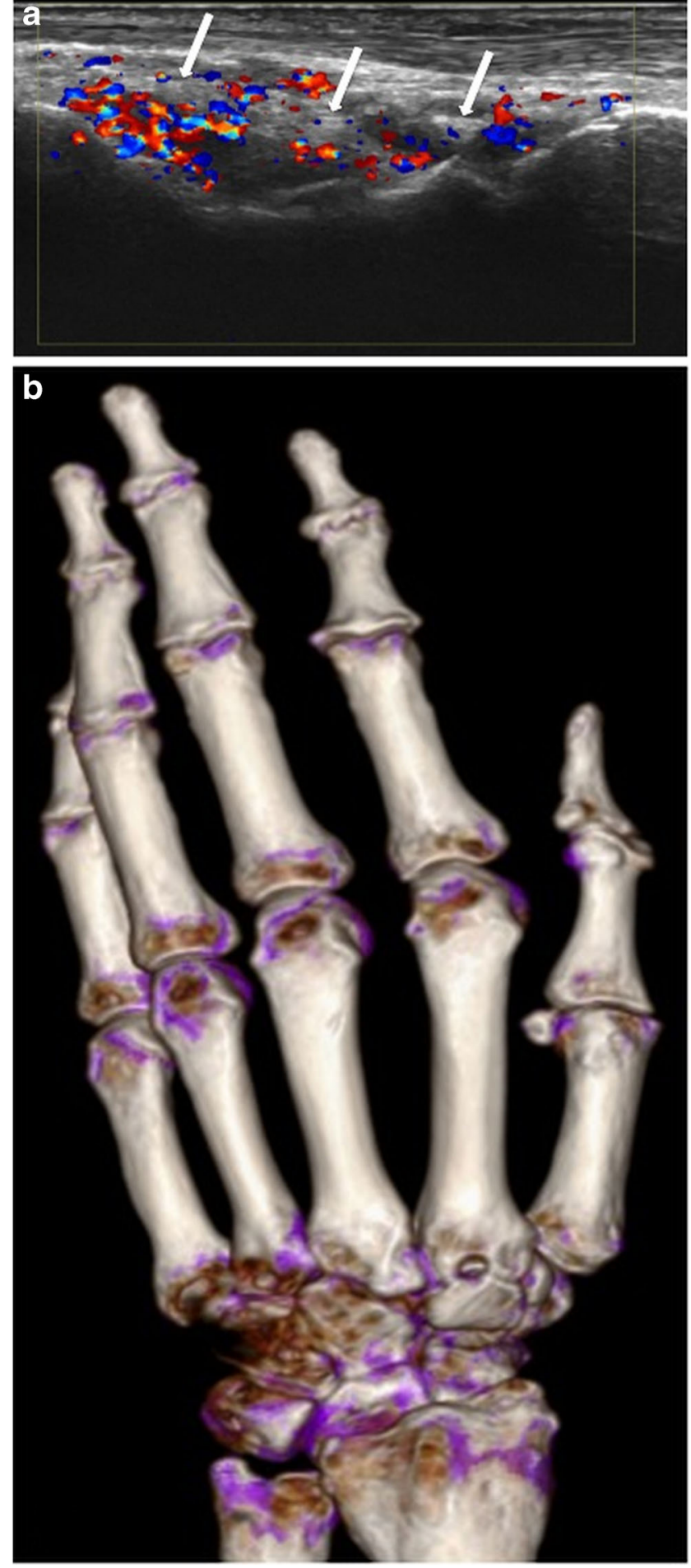

Fig. 3 Left hand of a 63-year-old male patient. a Longitudinal US scan of the dorsal wrist showing hypoechoic thickening, extensive hyperemia and echogenic deposits (white arrows) in RC and IC joints, rated as indeterminate. b DECT 3D volume rendered image of the same hand showing no MSU deposits, but severe osteoarthritis at RC, IC and carpometacarpal joints in terms of osteoarthritis 

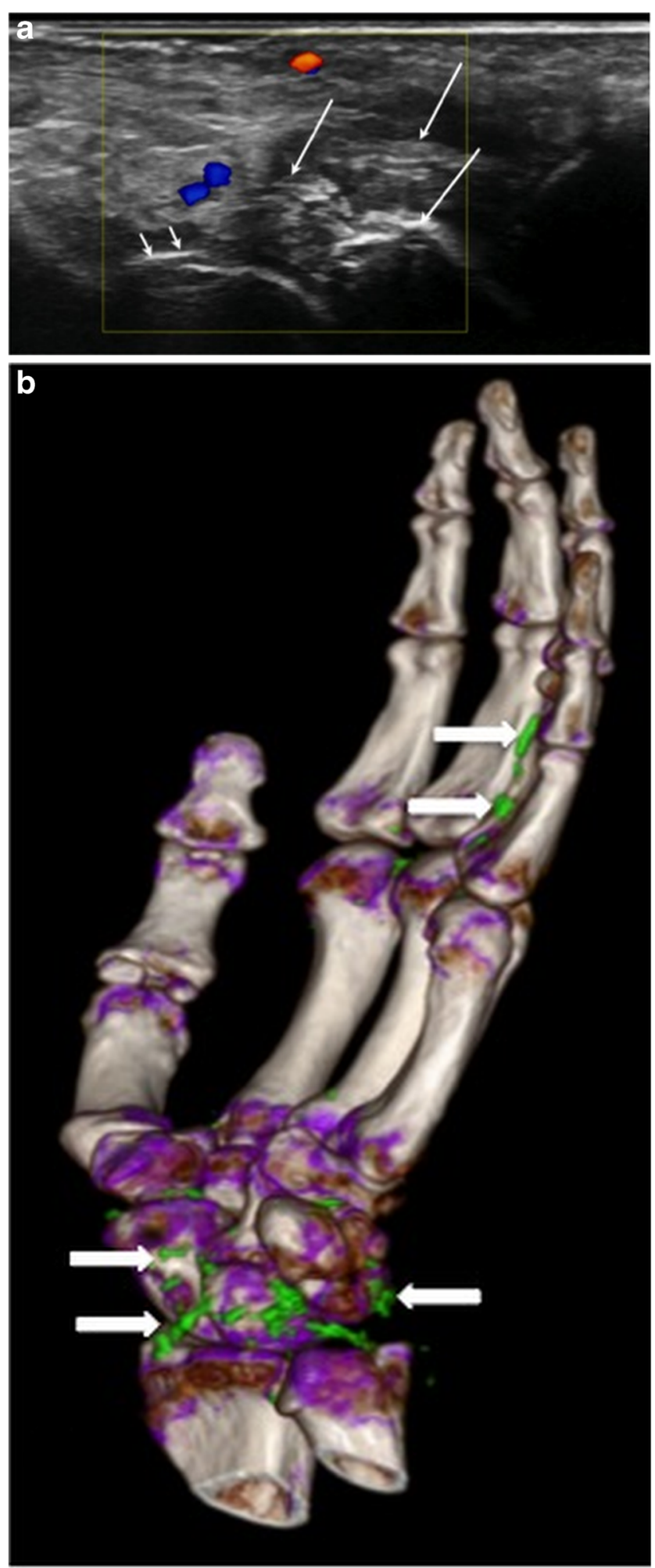

Fig. 4 Right hand of a 63-year-old male patient. a Longitudinal US scan over the volar wrist showing tophus formation (large arrows) and double contour (small arrows). b DECT 3D volume rendered image of the same hand showing MSU deposits at the wrist and flexor tendons

$(0.72-0.91)$ and specificity of $0.76(0.68-0.83)$ in a literature review of 11 studies. A recent study by Naredo et al. [33] showed a sensitivity of $84.6 \%$ and specificity of $83.3 \%$ for DCS in upper and lower limbs in a 12-structure US assessment. On the basis of these studies, the DCS has been incorporated into the ACR/EULAR guidelines [32].

As detailed in our results, 63 of the 66 patients with a positive DCS had evidence of MSU deposits on DECT. A phantom study by Diekhoff et al. [34] demonstrated that gouty deposits can reliably be detected by DECT at a concentration of $12.5 \%$, and CPPD at a concentration of $6.25 \%$, corresponding to deposits with mean Hounsfield unit values of 59.8 for MSU and 101.1 for CPPD [29]. For in vivo assessment the estimated lower limit of uric acid concentration per voxel has to be $35 \%$ or higher to be detectable by DECT $[4,32,35,36]$. It is possible that the three cases of positive DCS in our study with negative DECT findings may represent low concentration MSU disease or early disease with gouty deposits not yet visible on DECT. Furthermore, the presence of a DCS in our study population was related to an elevated mean uric acid level. Careful follow-up for further signs of gouty arthritis both clinically and by US is planned for these three subjects.

As recorded in our series, only $71 / 180$ patients (39.4\%) had a definitive diagnosis of gout with US versus $97 / 180$ patients $(53.9 \%)$ by DECT. Indeterminate US cases frequently had small deposits, suggesting that cases with less severe findings may be more challenging. Our study shows that osteoarthritis (OA) can be challenging in the US differential diagnosis, as $50.6 \%$ of the DECT negative patients were diagnosed to have OA with loose bodies by using coronal reformed CT images, and many of these patients were positive or indeterminate by US. US shows echogenic findings which may be secondary to calcium or MSU deposits, while DECT is capable of discriminating urate from non-urate compounds [22] (Figs. 3 and 4). This limitation of US was confirmed in our study (Table 2) as US showed fewer extra-articular gouty deposits (extensor tendon $22.5 \%$, flexor tendon $15.5 \%$ ) versus DECT (extensor tendon $30 \%$, flexor tendon 65\%). A prospective blinded randomized study by Choi et al. [35] showed a sensitivity of $84 \%$ and a specificity of $93 \%$ for detection of MSU deposits by DECT, but the most common location of MSU deposits in 20 evaluated patients were in lower extremities, and included only 6 hands/wrists out of 40 joints. The role of DECT has also been shown by Hu et al. [37] reporting a high sensitivity and specificity of $75.2 \%$ and $92.7 \%$ of DECT for the detection of gouty arthritis in upper and lower extremities. Zhu et al. [38] compared the diagnostic accuracy of DECT and US in various joints in 40 patients, also showing that DECT was superior to US in upper limbs, whereas no difference between the two methods was seen for the lower limbs. Finkenstaedt et al. [11] reported a change in the treatment plan in 23/43 patients with DECT, including 37 hands/wrists in their study.

Our study has several limitations. A major limitation of our study is that synovial fluid (SF) aspiration was not performed, because feasibility is limited in clinical practise [14, 21, 22]. 
Given the small dimensions of the hand/wrist joint recesses, fluid aspirations with small amounts of joint effusion often fail and are painful for the patients. Aspiration of MSU crystals may fail due to periarticular crystal depositions, or due to sedimentation of MSU with aspiration of crystal-free SF. Bongartz et al. [4] showed that in several patients with a negative SF analysis, DECT demonstrated evidence of MSU deposition in tendon sheaths and enthesial sites, suggesting that a significant number of patients could be tested false negative by SF examination alone [36]. Glazebrook et al. reported a sensitivity of $100 \%$ for DECT and a specificity of $79 \%$ and $89 \%$ (in two readers) for DECT compared to MSU positive joint aspiration results, but only 43 patients were evaluated and only 14 patients showed MSU deposits in wrists [16, 36]. Furthermore SF aspiration may not be possible if the patient refuses or is on anticoagulation. Given the difficulty of using SF analysis as a gold standard, US and DECT assume greater importance.

The evaluation of US and DECT by a single radiologist precludes our ability to evaluate inter-observer variations. Finally, we included patients with short duration of gout or a lesser degree of hyperuricemia, which may lead to MSU crystal deposition below the threshold of detection of DECT. The limit of detection of DECT is generally considered to be 2 $\mathrm{mm}$, so microscopic tophi may be missed [36]. A further limitation of our study is the high number of indeterminate cases by US ( 47 patients) limiting our specificity to $51 \%$, increasing to $96.4 \%$ when indeterminate cases are counted as negative. These cases seem particularly to benefit from DECT evaluation and should be addressed in further studies.

In summary the percentage of gouty deposits detected by US was significantly lower than that by DECT, especially in the extra-articular spaces.

Although the sensitivity of US for diagnosis of gouty hand and wrist arthritis is limited, it can be used as a first-line imaging modality in the presence of the DCS.

DECT was superior on overall detection and characterization, which might be of importance not only for diagnosis but for therapeutic follow-up in terms of delineation of gouty burden.

Acknowledgements Open access funding provided by University of Innsbruck and Medical University of Innsbruck.

Funding The authors state that this work has not received any funding.

\section{Compliance with ethical standards}

Guarantor The scientific guarantor of this publication is Andrea Klauser.

Conflict of interest The authors of this manuscript declare no relationships with any companies whose products or services may be related to the subject matter of the article.
Statistics and biometry One of the authors has significant statistical expertise (Prof. Ethan Halpern).

Informed consent Written informed consent was obtained from all patients in this study.

Ethical approval Institutional review board approval was obtained.

\section{Methodology}

- prospective

- performed at one institution.

Open Access This article is distributed under the terms of the Creative Commons Attribution 4.0 International License (http:// creativecommons.org/licenses/by/4.0/), which permits unrestricted use, distribution, and reproduction in any medium, provided you give appropriate credit to the original author(s) and the source, provide a link to the Creative Commons license, and indicate if changes were made.

\section{References}

1. Saag KG, Choi H (2006) Epidemiology, risk factors, and lifestyle modifications for gout. Arthritis Res Ther 1:2

2. Schueller-Weidekamm C, Schueller G, Aringer M, Weber M, Kainberger F (2007) Impact of sonography in gouty arthritis: comparison with conventional radiography, clinical examination, and laboratory findings. Eur J Radiol 62:437-434

3. Zhu Y, Pandya BJ, Choi HK (2011) Prevalence of gout and hyperuricemia in the US general population: the National Health and Nutrition Examination Survey 2007-2008. Arthritis Rheum 63: 3136-3141

4. Bongartz T, Glazebrook KN, Kavros SJ et al (2015) Dual-energy CT for the diagnosis of gout: an accuracy and diagnostid yield study. Ann Rheum Dis 74:1072-1077

5. Gentili A (2003) Advanced imaging of gout. Semin Musculoskelet Radiol 7:165-174

6. Grassi W, De Angelis R (2012) Clinical features of gout. Reumatismo 63:238-245

7. Harris MD, Siegel LB, Alloway JA (1999) Gout and hyperuricemia. Am Fam Physician 59:925-934

8. Dalbeth N, Doyle AJ (2012) Imaging of gout: an overview. Best Pract Res Clin Rheumatol 26:823-838

9. Dalbeth N (2013) Management of gout in primary care: challenges and potential solutions. Rheumatology 52:1549-1550

10. Zhang Z, Zhang X, Sun Y et al (2017) New urate depositions on dual-energy computed tomography in gouty arthritis during uratelowering therapy. Rheumatol Int 37:1365-1372

11. Finkenstaedt T, Manoliou A, Toniolo M et al (2016) Gouty arthritis: the diagnositc and therapeutic impact of dual-energy CT. Eur Radiol 26:3989-3999

12. Wu H, Xue J, Ye L, Zhou Q, Shi D, Xu R (2014) The application of dual-energy computed tomography in the diagnois of acute gouty arthritis. Clin Rheumatol 33:975-979

13. Modjinou DV, Krasnokutsky S, Gyftopoulos S et al (2017) Comparison of dual-energy CT, ultrasound and surface measurement for assessing tophus dissolution during rapid urate debulking. Clin Rheumatol 36:2101-2107

14. McQueen FM, Reeves Q, Dalbeth N (2013) New insights into an old disease: advanced imaging in the diagnosis and management of gout. Postgrad Med J 89:87-93 
15. Nicolaou S, Liang T, Murphy DT, Korzan JR, Ouellette H, Munk P (2012) Dual-energy CT: a promising new technique for assessment of the musculoskeletal system. AJR Am J Roentgenol 199:78-86

16. Glazebrook KN, Guimaraes LS, Murthy NS et al (2011) Identification of intraarticular and periarticular uric acid crystals with dual-energy CT: initial evaluation. Radiology 261:516-524

17. Manger B, Lell M, Wacker J, Schett G, Rech J (2012) Detection of periarticular urate deposits with dual energy $\mathrm{CT}$ in patients with acute gouty arthritis. Ann Rheum Dis 71:470-472

18. Huppertz A, Hermann KG, Diekhoff T, Wagner M, Hamm B, Schmidt WA (2014) Systemic staging for urate crystal deposits with dual-energy CT and ultrasound in patients with suspected gout. Rheumatol Int 34:763-771

19. Wallace SL, Robinson H, Masi AT, Decker JL, McCartey DJ, Yü TF (1977) Preliminary criteria for the classification of the acute arthritis of the primary gout. Arthritis Rheum 20:895-900

20. Neogi T, Jansen TL, Dalbeth N et al (2015) 2015 gout classification criteria: an American College of Rheumatology/European League Against Rheumatism collaborative initiative. Ann Rheum Dis 67: $2557-2568$

21. Gutierrez M, Schmidt WA, Thiele RG et al (2015) International consensus for ultrasound lesions in gout: results of Delphi process and web-reliability exercise. Rheumatology 54:1797-1805

22. Choi HK, Al-Arfaj AM, Eftekhari A et al (2009) Dual energy computed tomography in tophaceous gout. Ann Rheum Dis 68:16091612

23. Zhang W, Doherty M, Pascual E et al (2006) EULAR evidence based recommendations for gout. Part I: diagnosis. Report of a task force of the Standing Committee for International Clinical Studies Including Therapeutics (ESCISIT). Ann Rheum Dis 65:1301-1311

24. Mathieu S, Pereira B, Couderc M et al (2013) Usefulness of ultrasonography in the diagnosis of gout: a meta-analysis. Ann Rheum Dis $72: 23$

25. Chowalloor PV, Keen HI (2013) A systematic review of ultrasonography in gout and asymptomatic hyperuricemia. Ann Rheum Dis 72:638-645

26. Filippucci E, Riveros MG, Georgescu D, Salaffi F, Grassi W (2009) Hyaline cartilage involvement in patients with gout and calcium pyrophosphate deposition disease. Osteoarthritis Cartilage 17: 178-181

27. Wright SA, Filippucci E, McVeigh C et al (2007) High-resolution ultrasonography of the first metatarsal phalangeal joint in gout: a controlled study. Ann Rheum Dis 66:859-864

28. Thiele RG, Schlesinger N (2007) Diagnosis of gout by ultrasound. Rheumatology 46:1116-1121

29. Thiele RG (2011) Role of ultrasound and other advanced imaging in the diagnosis and management of gout. Curr Rheumatol Rep 13: 146-153

30. Pineda C, Amezcua-Guerra LM, Solano C et al (2011) Joint and tendon subclinical involvement suggestive of gouty arthritis in asymptomatic hyperuricemia: an ultrasound controlled study. Arthritis Res Ther 13:4

31. Ottaviani S, Allard A, Bardin T, Richette P (2011) An exploratory ultrasound study of early gout. Clin Exp Rheumatol 29:816-821

32. Ogdie A, Taylor WJ, Weatherall M et al (2015) Imaging modalities for the classification of gout: systematic literature review and metaanalysis. Ann Rheum Dis 74:1868-1874

33. Naredo E, Uson J, Jimenez-Palop M et al (2014) Ultrasounddetected musculoskeletal urate crystal deposition: which joints and what findings should be assessed for diagnosing gout? Ann Rheum Dis 73:1522-1528

34. Diekhoff T, Kiefer T, Stroux A et al (2015) Detection and characterization of crystal suspensions using single-source dual-energy computed tomography: a phantom model of crystal arthropathies. Invest Radiol 50:255-260

35. Choi HK, Burns LC, Shojania K et al (2012) Dual energy CT in gout: a prospective validation study. Ann Rheum Dis 71:14661471

36. Glazebrook KN, Kakar S, Ida CM, Laurini JA, Moder KG, Leng S (2012) False-negative dual-energy computed tomography in a patient with acute gout. J Clin Rheumatol 18:138-141

37. Hu HJ, Liao MY, Xu LY (2015) Clinical utility of dual-energy CT for gout diagnosis. Clin Imaging 39:880-885

38. Zhu $\mathrm{L}, \mathrm{Wu} \mathrm{H}, \mathrm{Wu} \mathrm{X}$ et al (2015) Comparison between dual-energy computed tomography and ultrasound in the diagnosis of gout of various joints. Acad Radiol 22:1497-1502 